\title{
Imaging Single ZnO Vertical Nanowire Laser Cavities using UV-laser Scanning Confocal Microscopy
}

\author{
Daniel J. Gargas, Maria Eugenia Toimil-Molares, Peidong Yang \\ Department of Chemistry, University of California, Berkeley, California 94720, and Materials Sciences Division, \\ Lawrence Berkeley National Laboratory, 1 Cyclotron Road, Berkeley, California 94720 \\ Email: p_yang@berkeley.edu
}

Semiconductor nanostructures have garnered considerable interest due to their unique optical properties and potential integration into optoelectonics and nanoscale devices. ${ }^{1}$ Among direct band gap materials, much attention has been given to $\mathrm{ZnO}$ due to its wide band gap (3.37 eV) and large exciton binding energy (60 $\mathrm{meV}$ ) at room temperature, as well as high thermal and mechanical stability. ${ }^{2}$ This has led to extensive research on the optical properties of $\mathrm{ZnO}$ nanowires, which have recently been demonstrated in solar cells, LEDs, and UV lasers. ${ }^{3-6}$ Here we report on the synthesis and characterization of single $\mathrm{ZnO}$ nanowire vertical cavities on their native growth substrate. Optimized growth conditions lead to diluted arrays of vertical $\mathrm{ZnO}$ nanowires, with spacing between wires varying between 2 and $50 \mu \mathrm{m}$. This geometrical arrangement enables the optical characterization of single vertical nanocavities by using a focused beamspot smaller than the distance between adjacent nanowires, as well as high-resolution photoluminescence imaging by UVlaser scanning confocal microscopy

Lasing has been observed in $\mathrm{ZnO}$ structures including micropillars, nanowires, and nanorods. ${ }^{3,-11}$ Investigation of the lasing characteristics of high density $\mathrm{ZnO}$ nanowire arrays has shown that high quality single-crystalline $\mathrm{ZnO}$ nanowires constitute ideal lasing nanocavities, which provide both a gain medium and a resonant cavity due to reflection at the planar endfacets. $^{12,} 13$ Lasing emission of a single nanowire placed horizontally on a substrate was first demonstrated in 2001 and subsequent work has developed a further understanding of the lasing mechanism in $\mathrm{ZnO}$ nanostructures. ${ }^{10,14-16}$ In most reports, nanowires were removed from their native sapphire substrate and transferred onto a blank $\mathrm{Si} / \mathrm{SiO}_{2}$ wafer for individual characterization, either mechanically using a micromanipulator or in a solution by sonication and drop-casting. While this process ensured an isolated environment for single nanowire characterization, it unfortunately rendered the nanowire in a horizontal geometry. In this geometrical arrangement, the nanowire is in direct contact with the substrate along its entire length, resulting in greater index matching and higher cavity losses. In addition, removal and transfer processes can damage the nanowire by introducing stress, defects, and localized fractures that result in further cavity losses. Damage to the planar end-facet connected to the growth substrate decreases the quality factor of the cavity. ${ }^{12}$

To avoid the drawbacks listed above for nanowires in a horizontal geometry, we turned our attention to vertical nanowires which have a greater refractive index than the surrounding air and, consequently, better mode confinement within the nanowire core. Unlike a horizontal nanowire, substrate properties such as dielectric constant and surface roughness have no effect on axial mode propagation within a vertical nanowire. It has been found that eliminating such adverse effects improves the lasing performance of a $\mathrm{ZnO}$ nanowire despite the decreased reflection occurring at the $\mathrm{ZnO}$-substrate end facet. ${ }^{10}$ Additionally, a vertical nanowire geometry provides greater directional coupling of far field PL emission into the collection mechanism of epifluorescent microscopy. By directly probing the $\mathrm{ZnO}$ vertical nanowires on their native substrate, we have avoided any adverse changes to nanowire structure that result from substrate removal and subsequent transfer to a horizontal geometry.

In addition, 3-dimensional (3D) mapping of the photoluminescence emission of single vertical nanowires was performed using UV-laser scanning confocal microscopy. This unique method of high-resolution microscopy combines a UV laser focused to a diffraction-limited spot $(\sim 220 \mathrm{~nm})$ with digital scanning electronics and a UV-enhanced detection system to image the PL emission from planar sections of a $\mathrm{ZnO}$ vertical nanowire from base to tip. The capability of this technique to selectively excite nanoscale regions of semiconductor nanowires in both horizontal and vertical geometries demonstrates a promising method for optically characterizing heterogeneous nanostructures for solar, LED, and optoelectonic applications.

Vertical $\mathrm{ZnO}$ nanowires were prepared on a-plane sapphire substrates decorated with low density of $\mathrm{Au}$ nanoparticles using a modified CVT method ${ }^{17}$. As previously reported for the synthesis of $\mathrm{Si}$ nanowires ${ }^{18}$, the density of nanowires is determined by the density of seeding Au catalyst nanoparticles on the substrate. Thus, instead of sputtering a $\mathrm{Au}$ thin film that results in a very dense arrays of nanowires, we dispersed low density of $\mathrm{Au}$ nanoparticles as metal catalysts by immersing the sapphire substrate first in a $10 \%$ aminopropyltriethoxysilane (APTES) solution for ten seconds and then in a diluted $\mathrm{Au}$ nanoparticle solution (Ted Pella, $80 \mathrm{~nm}$ diameter). After rinsing in DI water, the substrate was placed in a furnace with a 1:1 mixture of graphite and $\mathrm{ZnO}$ and heated to 900 ${ }^{\circ} \mathrm{C}$ for 30 minutes under constant Ar flow. Resulting diluted vertical nanowire arrays had local densities between $4 \times 10^{4}$ and $2 \times 10^{7}$ nanowires $/ \mathrm{cm}^{-2}$, corresponding to an inter-wire distance between 50 and $2 \mu \mathrm{m}$, respectively.

Stimulated emission in vertical nanowire cavities was generated by $266 \mathrm{~nm}$ excitation pulses from the fourth-harmonic of a Nd:YAG laser (Spectra-Physics, 8 ns pulse width, $10 \mathrm{~Hz}, 1-$ $50 \mu \mathrm{J} /$ pulse) focused onto the sample at a grazing angle of $16^{\circ}$ with respect to the substrate plane. PL emission was collected by a dark-field microscope objective (Nikon 50x, NA 0.55) and imaged by an EMCCD camera (Andor, iXon) or routed via multimode optical fiber coupled to a $0.3 \mathrm{~m}$ spectrometer equipped with a liquid nitrogen cooled CCD camera (PI Acton). The spectral resolution is $0.05 \mathrm{~nm}$ (1200 grooves/mm grating) or $0.1 \mathrm{~nm}(150$ grooves/mm grating). UV confocal microscopy was performed by coupling continuous wave, unpolarized $325 \mathrm{~nm}$ light from a HeCd laser (Melles Griot) into a home-built laser scanning 
confocal microscope equipped with an $\mathrm{x}-\mathrm{y}-\mathrm{z}$ piezoelectric stage (Physik Instrumente) and a UV-enhanced objective (Zeiss Ultrafluar, $100 \mathrm{x}$, NA 0.90 ). The laser beam was collimated prior to entering the back aperture of the objective and focused to a diffraction-limited spot onto a raster scanned sample. PL emission was collected back through the same objective and passed through a beamsplitter which routed half the PL signal to a UV-enhanced channel photo-multiplier (CPM) (Perkin-Elmer) for image construction, and half to a multi-mode optical fiber for spectral analysis described above. Residual laser light was removed by edge filters (Omega Optical). PL image construction was performed by a digital-to-analog controller (Digital Instruments, Nanoscope III) that synchronously plotted the TTL pulse count of the CPM while controlling the scanning motion of the piezoelectric stage. All measurements were performed at room temperature.

Figure 1a shows vertically oriented $\mathrm{ZnO}$ nanowires approximately $5-8 \mu \mathrm{m}$ in length and $80-130 \mathrm{~nm}$ in diameter. In contrast to dense nanowire arrays, several-micron distances between neighboring $\mathrm{ZnO}$ nanowires allow optical characterization of a single vertical nanowire without necessary transfer to a blank substrate. Thus, nanowire structure and geometry is preserved, which is important for investigating the unperturbed nature of nanowire photoluminescence, as well as potential device application. In addition, the isolated growth arrangement provides a suitable platform for elucidating the unique lasing properties of a single nanowire, such as Fabry-Perot lasing modes.

Longitudinal Fabry-Perot lasing modes of a $\mathrm{ZnO}$ nanowire cavity are schematically illustrated in Figure $1 \mathrm{~b}$ as three different wavelengths $\left(\lambda_{A}, \lambda_{B}, \lambda_{C}\right)$. Each wavelength satisfies an integer mode given by the interference equation $\mathrm{m} \lambda=2 \mathrm{~L}(n$ $\lambda d n / d \lambda)$, where $\mathrm{m}$ is the mode number, $\mathrm{L}$ is the cavity length of the nanowire, $n$ is the refractive index of $\mathrm{ZnO}$ at wavelength $\lambda$, and $d n / d \lambda$ is the Sellmeier first-order dispersion relation at $\lambda$. The Fabry-Perot modes of a single $\mathrm{ZnO}$ vertical nanowire cavity are observed in PL spectra collected at increasing pump energies, as shown in Figure 1c. The PL spectra (vertically offset for clarity) were collected at pump energy densities $\left(\mathrm{P}_{\mathrm{ex}}\right)$ of $383,536,980$, 1378,1454 , and $1760 \mu \mathrm{J} / \mathrm{cm}^{2}$. For $P_{\mathrm{ex}}$ less than $400 \mu \mathrm{J} / \mathrm{cm}^{2}$ the spectra are broad and featureless with a PL peak width of $15.9 \mathrm{~nm}$ (FWHM), which is consistent with spontaneous emission. However, at pump energies above $400 \mu \mathrm{J} / \mathrm{cm}^{2}$ the PL peak width decreases sharply to approximately $0.8 \mathrm{~nm}$ and the intensity increases significantly above the spontaneous emission background. This transition in PL emission is more clearly seen in a plot of total PL intensity vs $\mathrm{P}_{\mathrm{ex}}$. (Figure 1c inset graph) The sudden increase in slope of PL intensity versus pump energy, which occurs at roughly $400 \mu \mathrm{J} / \mathrm{cm}^{2}$, represents the transition from spontaneous to stimulated emission. This value agrees well with other reports on lasing of vertical $\mathrm{ZnO}$ nanowire arrays in which thresholds were reported between $120-500 \mu \mathrm{J} / \mathrm{cm}^{2}$ under ns pulsed excitation. ${ }^{19,20}$ The inset images in Figure 1c show a single $\mathrm{ZnO}$ vertical nanowire cavity under white-light illumination (top) and stimulated emission (bottom).

As discussed above, the sharp peaks in the PL spectra of Figure 1c correspond to Fabry-Perot lasing modes of the $\mathrm{ZnO}$ vertical nanowire cavity. The difference in peak position $\Delta \lambda$ (i.e. mode spacing) for a Fabry-Perot cavity is given by $\Delta \lambda=\lambda^{2}[2 \mathrm{~L}(n-$ $\lambda d n / d \lambda)]^{-1}$. Using reported values for $n(2.4)$ and $d n / d \lambda(-0.015$ $\left.\mathrm{nm}^{-1}\right)$ at $\lambda=387 \mathrm{~nm},{ }^{11}$ and $\mathrm{L}=8.1 \mu \mathrm{m}$ determined by SEM imaging, $\Delta \lambda$ is calculated at $1.53 \mathrm{~nm}$, which agrees well with the experimentally observed mode spacings of $1.6 \mathrm{~nm}$. The observed redshift in lasing modes at higher excitation pump energies is a result of bandgap renormalization with the semiconductor nanowire, which causes longer wavelengths to be preferentially amplified. $^{21}$

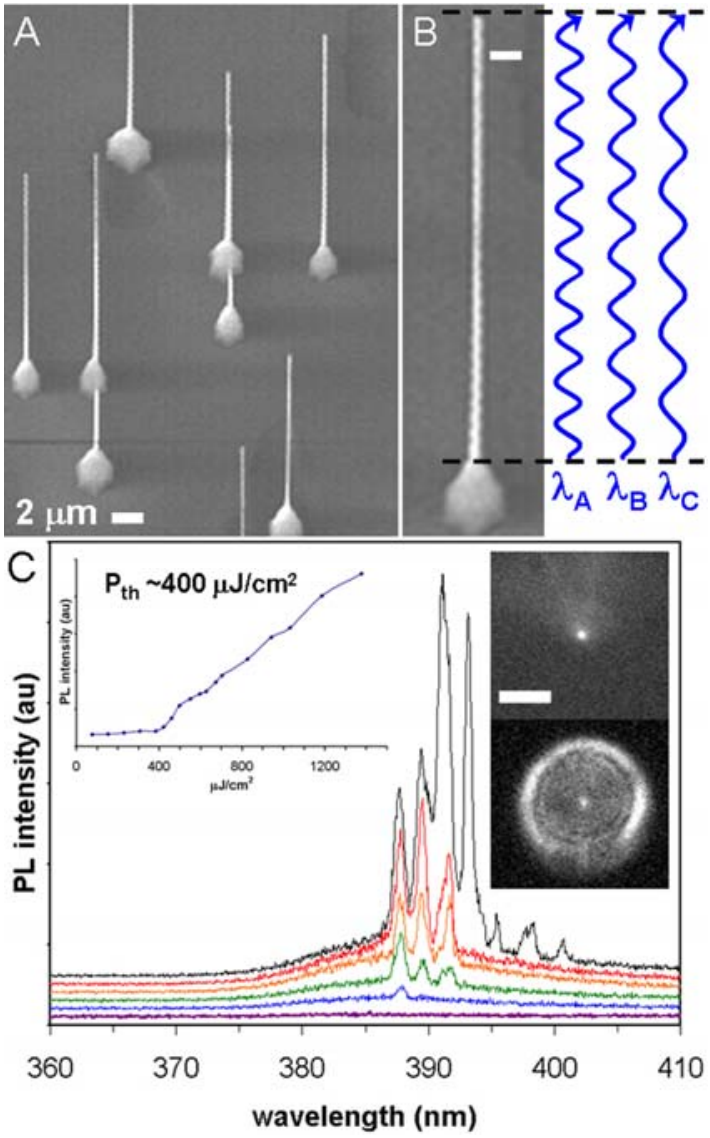

Figure 1. a) SEM image of $\mathrm{ZnO}$ vertical nanowire cavities grown on sapphire substrate. b) SEM image of single vertical nanowire with diagram showing Fabry-Perot lasing modes as wavelengths $\lambda_{\mathrm{A}}, \lambda_{\mathrm{B}}, \lambda_{\mathrm{C}}$. Scale bar is $300 \mathrm{~nm}$. c) Lasing spectra of single $\mathrm{ZnO}$ vertical nanowire cavity. Left inset: power dependence graph showing lasing threshold at roughly $400 \mu \mathrm{J} / \mathrm{cm}^{2}$. Right inset: dark-field scattering images of a $\mathrm{ZnO}$ vertical nanowire cavity from white light illumination (top) and lasing induced by $266 \mathrm{~nm}$ pulsed excitation (bottom). Scale bar is $2 \mu \mathrm{m}$.

To obtain high resolution optical imaging of individual $\mathrm{ZnO}$ vertical nanowires and map the spatial distribution of PL emission, UV-laser scanning confocal microscopy was utilized. Confocal microscopy takes advantage of the fact that light focused through a high numerical-aperture (NA) objective is reduced to a beamspot diameter of $0.61 * \lambda / \mathrm{NA}$. Thus, for $325 \mathrm{~nm}$ excitation the diffraction-limited beamspot has a diameter of $\sim 220$ $\mathrm{nm}$, which is small enough to excite individual nanowires of an array with inter-wire distances as small as $2 \mu \mathrm{m}$, and avoid residual PL emission from adjacent nanowires. Coupling this method of highly-selective spatial excitation to a microscope equipped with UV-sensitive detectors and scanning electronics, yields an imaging platform capable of high-resolution characterization of both spatial and spectral properties of photoluminescent nanostructures. In addition, the collected light can be mapped 3-dimensionally to provide a visual representation of the PL emission intensity by nanowire region.

A 3D mapping of the PL emission intensity from a single vertical nanowire on a sapphire substrate is shown in Figure 2a. The X- and Y- axes denote spatial position, whereas the Z- axis represents the PL emission intensity collected at a set 
planar position. In other words, the 3D image is a plot of the PL emission at each $\mathrm{X}$ - and $\mathrm{Y}$ - position on a fixed focal plane. Therefore, the vertical feature shown in Figure $2 \mathrm{a}$ is a representation of the band-edge PL emitted from an individual nanowire at a given focal plane. It should be noted that the $3 \mathrm{D}$ rendered PL image does not represent a measurement of nanowire height; rather it serves as a visual image of the total PL emission from a particular vertical section on the nanowire. The inset shows an SEM image of the $\mathrm{ZnO}$ nanowire cavity.
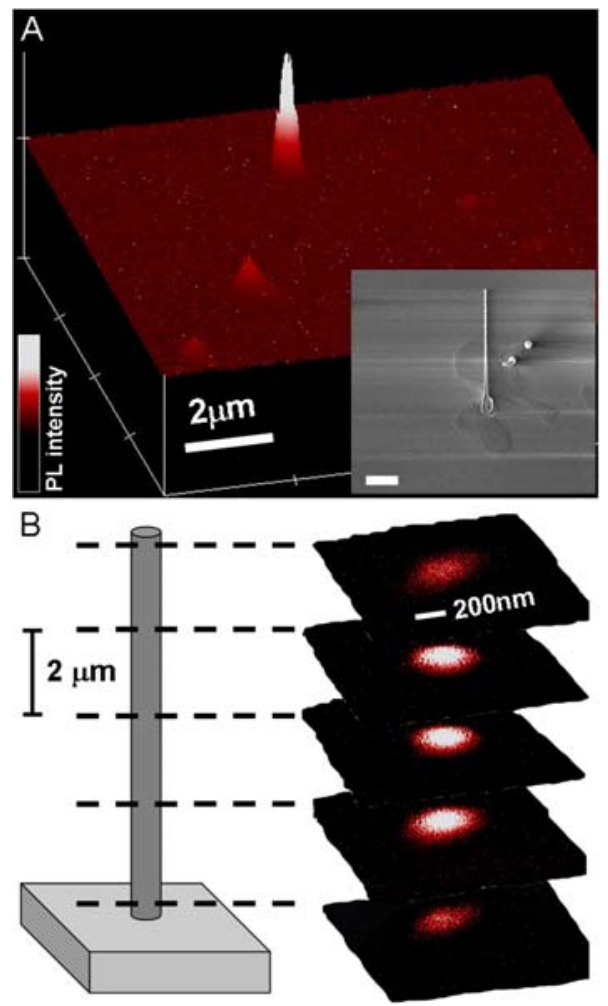

Figure 2. Vertically-sectioned PL imaging of a single $\mathrm{ZnO}$ vertical nanowire cavity. a) 3D-rendered confocal scanned PL image of $\mathrm{ZnO}$ vertical nanowire cavity. Inset: SEM image of $\mathrm{ZnO}$ vertical nanowire in (a). Scale bar is $2 \mu \mathrm{m}$. b) Diagram of $\mathrm{ZnO}$ vertical nanowire cavity with corresponding PL images collected from focal planes spaced $2 \mu \mathrm{m}$ apart vertically.

In addition to imaging vertical nanowires in the planar direction, the spatial selectivity of confocal microscopy was also utilized to image the PL emission by vertical sections of a nanowire. This was performed by raising or lowering the focal plane of the diffraction limited spot with respect to the sample during successive scans of an individual nanowire. Figure $2 b$ shows vertical sections of the nanowire imaged from base to tip by adjusting the z-position of the piezoelectric stage by $2 \mu \mathrm{m}$ after each PL emission scan. The images of each scan are stacked vertically next to a diagram of a vertical nanowire to show PL emission from each corresponding vertical section. These images in particular demonstrate the high resolution achieved by the confocal microscope and its ability to selectively image regions at the nanoscale. Cross-sectional analysis of the images shown in 2b, reveal a FWHM of approximately $320 \mathrm{~nm}$ (Figure S1), which is the combination of the laser point-spread function (e.g. focused beam diameter) and the vertical nanowire diameter $(\sim 100 \mathrm{~nm})$. The stacked images reveal a constant PL intensity throughout the height of the nanowire, which indicates the PL emission is spatial selective in both the planar and vertical dimensions. This technique could prove considerably useful for imaging submicron regions of a heterogeneous vertical nanowire structure.

In summary, the synthesis and optical characterization of individual $\mathrm{ZnO}$ vertical nanocavities is presented. Dilute arrays of $\mathrm{ZnO}$ vertical nanowires with inter-wire distances of $2-50 \mu \mathrm{m}$ were grown by a modified CVT method. This allowed optical characterization of single vertical nanowires by pulsed laser spectroscopy and UV-confocal microscopy. The mode structure of a single $\mathrm{ZnO}$ vertical nanowire cavity was observed and the lasing threshold was measured at $400 \mu \mathrm{J} / \mathrm{cm}^{2}$. Additional single nanowire characterization was performed by mapping the PL emission by region in both the planar and vertical dimensions, revealing height-selective imaging that had never before been presented on nanowire structures. This characterization method could provide highly-selective optical characterization of heteronanostructures emerging in the field of optoelectronics and nanophotonics that is unattainable by conventional microscopy.

Acknowledgment. This work was supported by the Director, Office of Basic Energy Sciences, Materials Sciences and Engineering Division, of the U.S. Department of Energy under Contract No. DE-AC02-05CH11231. The authors thank Steve Hoagland for assistance with instrument development, Taleb Mokari for assistance with electron microscopy, and Erik Garnett for discussions on synthesis. M.E.T.M thanks the MaxKade Foundation for financial support through a fellowship.

Supporting Information Available: Figure S1. This material is available free of charge via the internet at http://pubs.acs.org.

(1) Xia, Y. N.; Yang, P. D.; Sun, Y. G.; Wu, Y. Y.; Mayers, B.; Gates, B.; Yin, Y. D.; Kim, F.; Yan, Y. Q., Advanced Materials 2003, 15, 353.s

(2) Klingshirn, C.; Hauschild, R.; Fallert, J.; Kalt, H., Phys. Rev. B 2007, 75, (115203), 115203.

(3) Huang, M. H.; Mao, S.; Feick, H.; Yan, H. Q.; Wu, Y. Y.; Kind, H. Weber, E.; Russo, R.; Yang, P. D., Science 2001, 292, 1897.

(4) Law, M.; Greene, L. E.; Johnson, J. C.; Sayakally, R. J.; Yang, P. D., Nature Materials 2005, 4, 455.

(5) Lai, E.; Kim, W.; Yang, P. D., Nano. Res. 2008, 1, 123.

(6) Tsukazaki, A.; Ohtomo, A.; Onuma, T.; Ohtani, M.; Makino, T.; Sumiya M.; Ohtani, K.; Chichibu, S. F.; Fuke, S.; Segawa, Y.; Ohno, H.; Koinuma, H.; Dawasaki, M., Nature Materials 2005, 4, 42-46.

(7) Fallert, J.; Stelzl, F.; Zhou, H.; Reiser, A.; Thonke, K.; Sauer, R.; Klingshirn, C.; Kalt, H., Optics Express 2008, 16, (2), 1125-1131.

(8) Kwok, W. M.; Djurisic, A. B.; Leung, Y. J.; chan, W. K.; Phillips, D. L; Chen, H. Y.; Wu, C. L.; Gwo, S.; Xie, M. H., Chem. Phys. Lett 2005, 412, 141-144

(9) Yan, H. Q.; Johnson, J. C.; Law, M.; He, R.; Knutsen, K. P.; McKinney, J. R.; Pham, J.; Saykally, R. J.; Yang, P., Adv. Mater. 2003, 15, 1907.

(10) Johnson, J. C.; Yan, H.; Schaller, R. D.; Haber, L. H.; Sayakally, R. J. Yang, P., Journal of Physical Chemistry B 2001, 105, (46), 11387-11390.

(11) Zimmler, M. A.; Bao, J.; Capasso, F.; Müller, S.; Ronning, C., Applied Physics Letters 2008, 93, 051101.

(12) Maslov, A. V.; Ning, C. Z., Applied Physics Letters 2003, 83, (6), 1237 1239.

(13) Vugt, L. K. v.; Rühle, S.; Vanmaekelbergh, D., Nano Letters 2006, 6 , 2707.

(14) Johnson, J. C.; Yan, H.; Yang, P.; Sayakally, R. J., Journal of Physical Chemistry C 2003, 107, 8816-8828.

(15) Song, J. K.; Willer, U.; Szarko, J. M.; Leone, S. R.; Li, S.; Zhao, Y., Journal of Physical Chemistry C 2008, 112, 1679-1684.

(16) Johnson, J. C.; Knutsen, K. P.; Yan, H. Q.; Law, M.; Zhang, Y.; Yang, P.; Saykally, R. J., Nano. Lett. 2004, 4, 197-204.

(17) Yan, H. Q.; He, R. R.; Johnson, J. C.; Law, M.; Sayakally, R. J.; Yang, P. D., J. Am. Chem. Soc. 2003, 125, 4728.

(18) Hochbaum, A. I.; Fan, R.; R., H.; Yang, P. D., Nano Letters 2005, 5, 457.

(19) Özgür, Ü.; Alivov, Y. I.; Liu, C.; Teke, A.; Reshchikov, M. A.; Doğan S.; Avrutin, V.; Cho, S.-J.; Morkoç, H., Journal of Applied Physics 2005, 98, 041301.

(20) Sirbuly, D. J.; Law, M.; Yan, H.; Yang, P., Journal of Physical Chemistry B 2005, 109, 15190-15213.

(21) Zhou, H.; Wissinger, M.; Fallert, J.; Hauschild, R.; Stelzl, F.; Klingshirn, C.; Kalt, H., Applied Physics Letters 2007, 91, 181112 

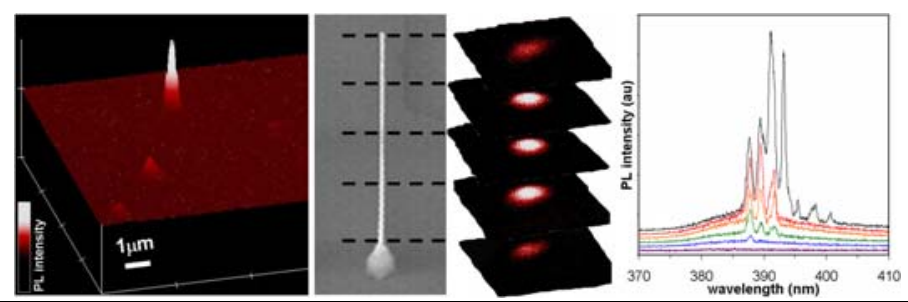

We report the fabrication and optical characterization of individual ZnO vertical nanowire laser cavities. Dilute nanowire arrays with inter-wire spacing $>10 \mu \mathrm{m}$ were produced by a modified chemical vapor transport (CVT) method yielding an ideal platform for single nanowire imaging and spectroscopy. Lasing characteristics of a single vertical nanowire are presented, as well as high-resolution photoluminescence imaging by UV-laser scanning confocal microscopy. In addition, 3-dimensional (3D) mapping of the photoluminescence emission performed in both planar and vertical dimensions demonstrates height-selective imaging useful for vertical nanowires and hetero-nanostructures emerging in the field of optoelectronics and nanophotonics. 


\section{DISCLAIMER}

This document was prepared as an account of work sponsored by the United States Government. While this document is believed to contain correct information, neither the United States Government nor any agency thereof, nor The Regents of the University of California, nor any of their employees, makes any warranty, express or implied, or assumes any legal responsibility for the accuracy, completeness, or usefulness of any information, apparatus, product, or process disclosed, or represents that its use would not infringe privately owned rights. Reference herein to any specific commercial product, process, or service by its trade name, trademark, manufacturer, or otherwise, does not necessarily constitute or imply its endorsement, recommendation, or favoring by the United States Government or any agency thereof, or The Regents of the University of California. The views and opinions of authors expressed herein do not necessarily state or reflect those of the United States Government or any agency thereof or The Regents of the University of California. 